\title{
Maximally Entangled States of Four Nonbinary Particles
}

\author{
Mario $\mathrm{Gaeta}^{a}$, Andrei Klimov ${ }^{a}$, and Jay Lawrence ${ }^{b, c}$ \\ (a) Department of Physics, University of Guadalajara, Guadalajara, Jalisco, Mexico, \\ (b) Department of Physics and Astronomy, \\ Dartmouth College, Hanover, NH 03755, USA and \\ (c) The James Franck Institute, University of Chicago, Chicago, IL 60637, USA
}

(Dated: revised April 19, 2022)

\begin{abstract}
Systems of four nonbinary particles, each particle having $d \geq 3$ internal states, exhibit maximally entangled states that are inaccessible to four qubits. This breaks the pattern of two- and threeparticle systems, in which the existing graph states are equally accessible to binary and nonbinary systems alike. We compare the entanglement properties of these special states (called $P$-states) with those of the more familiar GHZ and cluster states accessible to qubits. The comparison includes familiar entanglement measures, the "steering" of states by projective measurements, and the probability that two such measurements, chosen at random, leave the remaining particles in a Bell state. These comparisons demonstrate not only that $P$-state entanglement is stronger than the other types, but that it is maximal in a well-defined sense. We prove that GHZ, cluster, and $P$-states represent all possible entanglement classes of four-particle graph states with prime $d \geq 3$.

PACS numbers: 03.67.Mn, 03.65.Ud, 03.65.Aa
\end{abstract}




\section{INTRODUCTION}

This work is motivated by the general question of how multipartite entanglement develops as both the number of particles and the internal dimension of each particle increase. Our focus here is on the observation that the four particle system represents a sort of transitional case, not only because more than a single type of graph state [1] becomes accessible, but more importantly, because the number of such types increases (from two to three) on going from the binary to the nonbinary cases (all prime $d \geq 3$ ). The term graph state is used here to refer to any nonseparable eigenstate of Pauli operators, including those of only two or three particles. So, for example, two-particle systems exhibit generalized Bell states [2]4], and three-particle systems exhibit generalized GHZ states [5], in both cases for any $d$, with these being the only graph state options. The situation changes for four-particle systems, first because qubit systems can exhibit cluster states $(C)$ as well as GHZ states $(G)$, and second, because nonbinary systems (of any prime $d \geq 3$ ) can access a third type of graph state, called $P$. The $P$ states were discovered in the search for complete sets of mutually unbiased bases (MUBs) for four qudits [5]. Here we document the entanglement properties of the $P$ states in detailed comparisons with $G$ and $C$ states, and prove that no other types of graph states exist for four particles of any prime $d$.

Further motivation is provided by recent ideas for characterizing multipartite entanglement [6, 7] of pure states through the set of reduced density operators associated with all bipartitions; or equivalently, the mixed states of all subsystems of up to half the size of the whole system. If all one-particle subsystems are maximally mixed, then the state is called 1-MM [7], ..., if all $k$-particle subsystems are maximally mixed, then it is called $k$-MM. If a state $|\psi\rangle$ is $k$-MM for $k=[N / 2]$ (the integer part of $N / 2$ ), then $|\psi\rangle$ is a "maximally multipartite entangled state (MMES)" [6]. The following curious situation exists for qubits [7]: MMES states exist for $N=2,3,5$, and 6 qubits, while none exists for $N=4$ or any $N \geq 8$ (with the case of $N=7$ unresolved). A central result of this paper is that the $P$ states are MMES for all prime $d$, while the $G$ and $C$ states fall short for all $d$; the $P$ states (alone among graph states) fill a kind of gap that would otherwise exist for four-particle systems.

We confine our discussion here to graph states because of their prominent place in practical as well as foundational pursuits, as well as their natural extensions to nonbinary systems. 
There have been extensive studies of entanglement in many-qubit systems [8], stimulated in particular by the introduction of cluster states and more general graph states [1], with their potential for measurement-based quantum computation [9, 10]. The property of maximal connectedness, by which graph states can be steered selectively into entangled final states, was introduced in Ref. [1]. The entanglement properties of many-qudit systems $(d \geq 3)$ has also received much attention [4, 15, 11, 12], and the advantages of these nonbinary systems for quantum communication have been pointed out [13 15] and demonstrated [16]. The stabilizer formalism for graph states has been generalized to nonbinary systems [17-[19], with useful connections to mutually unbiased measurements [4, 20], which we shall exploit here.

The plan of the paper is as follows: In the next section we write out the graph states explicitly and reduce these to their simplest forms, which define the Schmidt measures. The reduced forms are used in Sec. III to evaluate entanglement measures, and Sec. IV presents an analysis of the steering of states by projective measurements utilizing the stabilizer formalism. While the above discussions are self-contained, Appendix A presents a more formal approach based on the adjacency matrix, which is then employed in Appendices B and C to prove that GHZ, cluster, and $P$-states represent all distinct entanglement classes for four-qudit graph states with prime $d$. The concluding Sec. V summarizes the results and discusses their implications for remaining unanswered questions.

\section{GRAPH STATES OF FOUR NONBINARY PARTICLES}

Of all graphs which may be written down for four-qudit systems, only three represent distinct entanglement classes, as we prove in Appendices B and C. The simplest graphs representative of each class are shown in Fig. 1. There is a three-sided alternative for $C$ states, but we declare the four-sided graph to be simpler because of its higher symmetry.

The states are constructed directly from the graphs according to the following wellknown rules. One sums over all $d^{4}$ elements of the standard product basis, $|i, j, k, l\rangle \equiv$ $|i\rangle \otimes|j\rangle \otimes|k\rangle \otimes|l\rangle$, associating each index with a graph vertex. Each term in the sum is weighted by phase factor dictated by the graph edges. In our examples a blue edge connecting points $i$ and $j$ contributes a phase factor $\omega^{i j}[$ with $\omega \equiv \exp (2 \pi i / d)]$, while a red 
4

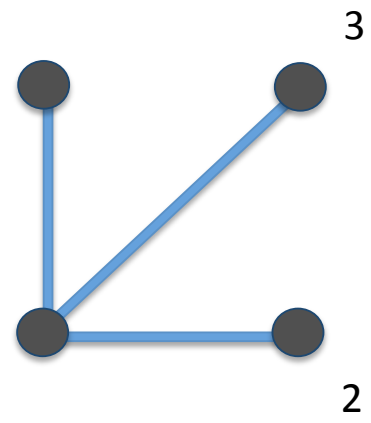

G

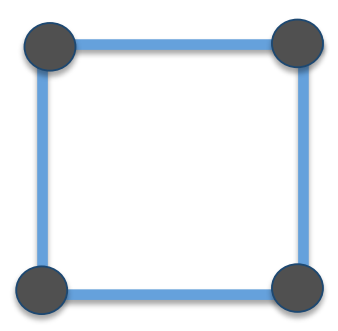

C

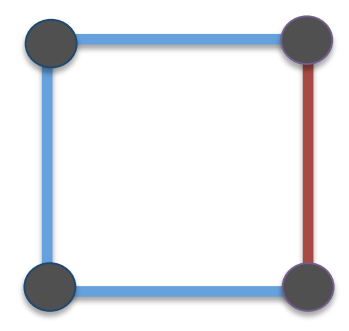

$P$

FIG. 1: Graphs defining the four-particle GHZ (G), cluster (C), and P states. Blue edges dictate $\omega^{i j}$ factors, red dictate $\omega^{-i j}$.

edge contributes $\omega^{-i j}$. Accordingly, the $G$ state is

$$
|G\rangle=d^{-2} \sum_{i, j, k, l}|i, j, k, l\rangle \omega^{i(j+k+l)},
$$

with all four sums extending from 0 to $d-1$. The $C$ state is

$$
|C\rangle=d^{-2} \sum_{i, j, k, l}|i, j, k, l\rangle \omega^{j(i+k)+l(k+i)}
$$

and the $P$ state, differing by the color of a single edge, is

$$
|P\rangle=d^{-2} \sum_{i, j, k, l}|i, j, k, l\rangle \omega^{j(i-k)+l(i+k)}
$$

Remarkably, the minus sign strengthens the entanglement for all $d$ except 2 , where it simply reproduces the $|C\rangle$ state.

These expressions can be simplified immediately by recognizing internal Fourier transforms. Recall the one-qudit operators,

$$
Z=\sum_{k=0}^{d-1}|k\rangle \omega^{k}\langle k| \quad \text { and } \quad X=\sum_{k=0}^{d-1}|k+1\rangle\langle k|
$$

these define the one-qudit states $|k\rangle$ as eigenstates of $Z$ with eigenvalues $\omega^{k}$, and their Fourier-transforms,

$$
\frac{1}{\sqrt{d}} \sum_{0}^{d-1}|k\rangle \omega^{j k} \equiv|j\rangle_{x}
$$


as eigenstates of $X$ with eigenvalues $\omega^{-j}$. Considering first the GHZ expression (1), one recognizes the sums over $j, k$, and $l$ as generating one-qudit states $|i\rangle_{x}$ for each of qudits 2 , 3 , and 4 . Now, locally transforming each of these $X$ states to $Z$ states, one obtains

$$
\left|G^{\prime}\right\rangle=\frac{1}{\sqrt{d}} \sum_{i}|i, i, i, i\rangle,
$$

where the prime denotes local equivalence to $|G\rangle$ (Eq. 1). Similar considerations apply to the $C$ and $P$ expressions, Eqs. 2 and 3 , although now only two of the sums may be interpreted as Fourier transforms, not three. Thus choosing the $j$ and $l$ sums, the second and fourth qudits appear in $X$-states with the arguments appearing below. Locally transforming these to $Z$-states, one obtains

$$
\begin{aligned}
\left|C^{\prime}\right\rangle & =\frac{1}{d} \sum_{i, k}|i, i+k, k, i+k\rangle . \\
\left|P^{\prime}\right\rangle & =\frac{1}{d} \sum_{i, k}|i, i-k, k, i+k\rangle .
\end{aligned}
$$

It is remarkable that the last two states take the general form

$$
|\psi(\gamma)\rangle=\frac{1}{d} \sum_{i, k}|i, i+\gamma k, k, i+k\rangle,
$$

and that the states with $\gamma=0,1$ belong to the same entanglement class $\{C\}$, while the $\{P\}$ class contains all others, $\gamma=2,3, \ldots, d-1$. This is proved in Appendices B and C.

The final expressions (6-8) cannot be simplified further, and thus make obvious a couple of simple entanglement measures: The Schmidt measure, $M_{S}$, refers to the minimum number of terms, $N_{\min }(\psi)$ in a separable basis expansion of $|\psi\rangle$ [21]. It is defined for nonbinary systems by

$$
M_{S}=\log _{d} N_{\min }(\psi),
$$

and takes the values 1,2 , and 2 for $G, C$, and $P$ states, respectively. Closely related is the Pauli persistency $(P P)$, defined as the minimum number of one-particle Pauli measurements required to completely remove the entanglement [1]. While in general $P P$ is an upper bound on $M_{S}$ [8], it is easy to verify that in our cases (6-8) the two are identical. These simplest measures fail to distinguish between the $C$ and $P$ states, but a more quantitative generalized response to Pauli measurements (Sec. IV) shows a dramatic difference.

We should also remark that equations 6-8 reveal symmetry properties not obvious from the graphs themselves: The GHZ and $P$ states are symmetric under the interchange of any 
two qudits and the entanglement is uniformly distributed over all pairs, while the cluster states are less symmetric - the 2-4 pair is not equivalent to 1-4 or 3-4. It is equivalent to the 1-3 pair, as one can show by Fourier transforming all particles, with the result that 1 and 3 acquire the repeated index in place of 2 and 4 . The physical consequence of this asymmetry is that particles are more entangled with their nearest neighbors on the square graph than with the diagonally-coordinated particle. This concentration of the entanglement has been understood to make it more robust than GHZ entanglement [1]. The remarkable thing about the $P$ states is that they share the uniform entanglement distribution of GHZ states, but with enhanced robustness exceeding that of the $C$ states. These properties will be demonstrated quantitatively in the next two sections.

\section{ENTANGLEMENT}

A thorough yet reasonably concise comparison of entanglement properties is afforded by the approach of Facchi et. al. [6] and Arnaud and Cerf [7], which focuses on the reduced density matrices $\rho_{A}$ and purities $\pi_{A}=\operatorname{Tr}_{A} \rho_{A}^{2}$ [or equivalently, the linear entropies $\sim\left(1-\pi_{A}\right)$ ] of all subsystems $(A)$. We evaluate these quantities for each of the three states and, at the end, comment on the concurrence and wedge product measures, which are both functions of $\pi_{A}$.

To begin, consider arbitrary bipartitions of pure four-particle states into subsystems $A$ and $B$. The Hilbert space is thus factorized, $\mathcal{H}=\mathcal{H}_{A} \otimes \mathcal{H}_{B}$, with subsystem dimensions $\left(D_{A}, D_{B}\right)$ being $\left(d, d^{3}\right)$ for the 1-3 partitions and $\left(d^{2}, d^{2}\right)$ for the 2-2 partitions. A general pure state of the system is expanded in the tensor product basis (as in Ref. [22]),

$$
|\psi\rangle=\sum_{a=1}^{d_{A}} \sum_{b=1}^{d_{B}} c_{a, b}|a\rangle_{A} \otimes|b\rangle_{B},
$$

where $\left\{|a\rangle_{A}\right\}$ and $\left\{|b\rangle_{B}\right\}$ comprise orthonormal bases in $\mathcal{H}_{A}$ and $\mathcal{H}_{B}$, respectively. The summation over $b$ at fixed $a$ identifies the particular state in B, called $\left|\psi_{a}\right\rangle_{B}$, that is associated with the basis state $|a\rangle_{A}$ in $A$. The set of all such "associated states," both those in $B$ and their counterparts in $A$, are defined implicitly by the expressions

$$
|\psi\rangle=\sum_{a}|a\rangle_{A}\left|\psi_{a}\right\rangle_{B}=\sum_{b}\left|\psi_{b}\right\rangle_{A}|b\rangle_{B}
$$


where obviously $\left|\psi_{a}\right\rangle_{B}$ and $\left|\psi_{b}\right\rangle_{A}$ are not normalized. Defining the reduced density matrices for parts $A$ and $B$ in the usual manner, $\rho_{A}=\operatorname{Tr}_{B}|\psi\rangle\langle\psi|$ and $\rho_{B}=\operatorname{Tr}_{A}|\psi\rangle\langle\psi|$, and taking the traces in the bases $\left\{|b\rangle_{B}\right\}$ and $\left\{|a\rangle_{A}\right\}$, respectively, one obtains

$$
\left\langle a\left|\rho_{A}\right| a^{\prime}\right\rangle_{A}=\left\langle\psi_{a^{\prime}} \mid \psi_{a}\right\rangle_{B} \quad \text { and } \quad\left\langle b\left|\rho_{B}\right| b^{\prime}\right\rangle_{B}=\left\langle\psi_{b^{\prime}} \mid \psi_{b}\right\rangle_{A}
$$

that is, the reduced density matrix of $A$ is identical to the overlap matrix of the associated states in $B$, and vice-versa. Subsystem $A$ is maximally mixed if and only if

$$
\rho_{A}=\mathcal{I}_{A} / D_{A} \quad \text { and hence } \quad \pi_{A}=1 / D_{A}, \quad \text { (maximal mixing) }
$$

where it is understood that $D_{A} \leq D_{B}$ (since $\pi_{A}=\pi_{B}$, as follows from 13 ).

Let us now evaluate $\rho_{A}$ for all subsystems consisting of one particle (we write $A=n$, where $n=1-4$ denotes which particle), and then two particles $(A=n, m)$. Regarding first the one-particle cases, it is a well-known property of graph states that all one-particle subsystems are maximally mixed, so that

$$
\rho_{n}=\mathcal{I}_{n} / d \quad \Rightarrow \quad \operatorname{Tr}_{n} \rho_{n}^{2}=1 / d
$$

This result is easily confirmed by noting that in all cases, the associated states $\left\{\left|\psi_{a}\right\rangle_{B}\right\}$ form orthonormal bases (apart from the factors of $1 / \sqrt{d}$ ) in subspaces of $\mathcal{H}_{B}$ of dimension $d$.

For the two-particle subsystems $(A=n, m)$, we proceed on a case-by-case basis. We begin with the $P$ states, which (ironically) provide the simplest case. Choose $A=1,3$ for simplicity. With each basis state $|i, k\rangle$ in $A$, there is a unique associated state $\left|\psi_{i, k}\right\rangle=$ $|i-k, i+k\rangle / d$ in $B$, so long as $d$ is a prime. These associated states form an orthonormal basis (except for the $1 / d$ factors) in $B$, of dimension $d^{2}$. According to Eq. $13, \rho_{n, m}(P)$ is proportional to the identity on $\mathcal{H}_{A}$, or more explicitly,

$$
\left\langle i j\left|\rho_{n, m}(P)\right| i^{\prime} j^{\prime}\right\rangle=\delta_{i i^{\prime}} \delta_{j j^{\prime}} / d^{2} \quad \Rightarrow \quad \operatorname{Tr}_{n, m} \rho_{n, m}^{2}(P)=1 / d^{2}
$$

This result holds for any choice of the pair $(n, m)$ and it shows that all such pairs are in maximally mixed states. This property makes $P$ states MMES, unique among four-qudit states. Let us proceed to see how the other cases fall short.

The $G$ states are less entangled from the current perspective because the coefficients $c_{a b}$ in Eq. 11 vanish unless the two indices are the same, so that the effective basis states in $A$ are $|i i\rangle_{n, m}$, and the associated states in $B$ are $\left|\psi_{i i}\right\rangle_{B}=|i i\rangle_{p, q} / \sqrt{d}$, where $(n, m)$ and $(p, q)$ 
TABLE I: Values of $\pi_{A} \equiv \operatorname{Tr}_{A} \rho_{A}^{2}$ for all one and two-particle subsystems $(A)$ of four particles in pure $G, C$, and $P$ states. Maximal mixing is indicated by minimum values: $1 / d$ for one-particle subsystems and $1 / d^{2}$ for two-particle subsystems.

\begin{tabular}{|c|c|c|c|}
\hline State & $A=n$ & $n, n+2$ & $n, n \pm 1$ \\
\hline$G$ & $1 / d$ & $1 / d$ & $1 / d$ \\
$C$ & $1 / d$ & $1 / d$ & $1 / d^{2}$ \\
$P$ & $1 / d$ & $1 / d^{2}$ & $1 / d^{2}$ \\
\hline
\end{tabular}

label any two distinct pairs. Clearly the associated states form an orthonormal set (except for the $1 / \sqrt{d}$ factor), but they span only a $d$-dimensional subspace of $B$. So, although Eq. 13 reads concisely as $\left\langle a\left|\rho_{n, m}(G)\right| a^{\prime}\right\rangle=\delta_{a a^{\prime}} / d$, a more explicit reference to the full subspace $\mathcal{H}_{A}$ reads

$$
\left\langle i j\left|\rho_{n, m}(G)\right| i^{\prime} j^{\prime}\right\rangle=\delta_{i i^{\prime}} \delta_{j j^{\prime}} \delta_{i j} / d \quad \Rightarrow \quad \operatorname{Tr}_{n, m} \rho_{n, m}^{2}(G)=1 / d
$$

This shows that $\rho_{n, m}(G)$ is not maximally mixed because the effective subsystem dimension is less than $D_{A}$. Results thus far are collected in Table I.

The $C$ states are more complicated because the choice of pairs matters. Consider first $A=1,2$ : With every basis state $|i, i+k\rangle$ in $A$, there is a unique associated state $|k, i+k\rangle / d$ in $B=3,4$. The latter form an orthonormal basis of dimension $d^{2}$, so that, as with $P$ states, $\rho_{1,2}(C)$ is proportional to the identity on $\mathcal{H}_{A}$. The identical situation clearly arises for another pair, namely $A=1,4$, so that these two pairs are maximally mixed:

$$
\rho_{1,2}(C)=\rho_{1,4}(C)=\rho_{n, m}(P)
$$

The remaining pair is $n, m=1,3$, or equivalently 2,4 . It is simpler to identify the latter with $A$, because clearly its effective dimension is only $d$. So, given the basis state $|j j\rangle$ in A (where $j \equiv i+k)$, the associated state in $B$ is $\left|\psi_{j j}\right\rangle=\sum_{i}|i, j-i\rangle_{B} / d$. The latter clearly form an orthonormal set except for the overall constant; evaluating the orthogonality matrix, one finds $\left\langle j j\left|\rho_{2,4}(C)\right| j^{\prime} j^{\prime}\right\rangle=\left\langle\psi_{j j} \mid \psi_{j^{\prime} j^{\prime}}\right\rangle_{B}=\delta_{j j^{\prime}} / d$, which is identical to the GHZ case leading to Eq. 17, and so

$$
\rho_{2,4}(C)=\rho_{n, m}(G)
$$

Equations 18 and 19 show that while nearest neighbor pairs on the square are maximally mixed, diagonally coordinated pairs are not. 
In summary, as shown on Table I, all one-particle subsystems $(n)$ are maximally mixed, so that $G, C$, and $P$ states are all 1-MM. Two-particle subsystems show a steady progression, but only the $P$ states achieve 2-MM. Related entanglement measures take their maximum values in this case.

\section{A. Related entanglement measures}

We comment briefly on the concurrence and the wedge product measure: Although these provide different information about mixed states, and may evolve differently under dissipative evolution [22], they reduce to functions of the purity when applied to bipartite partitions of pure states.

The concurrence was first introduced [23] in the context of pure states of two qubits, generalized [24] to mixed states of two qubits, and further generalized [25, 26] to bipartite partitions of systems of arbitrary dimension. It was shown that in the case of pure states, the concurrence of a bipartition $(A-B)$ reduces (within an arbitrary multiplicative constant) to [26]

$$
\mathcal{C}=\sqrt{1-\pi_{A}},
$$

noting that $\pi_{A}=\pi_{B}$. So the minimal entries in Table I represent maximal values of $\mathcal{C}$.

The wedge product is a measure of the orthogonality of two associates states; its square is defined as

$$
\mathcal{W}^{2}\left(a, a^{\prime}\right)=\left\langle\psi_{a} \mid \psi_{a}\right\rangle_{B}\left\langle\psi_{a^{\prime}} \mid \psi_{a^{\prime}}\right\rangle_{B}-\left|\left\langle\psi_{a} \mid \psi_{a^{\prime}}\right\rangle_{B}\right|^{2},
$$

that is, $\mathcal{W}$ is the product of norms times the sine of the angle between the two vectors in the subspace of $\mathcal{H}_{B}$ that they span. An entanglement measure was first introduced [27] as an average of $\mathcal{W}^{2}$ over all one-particle subsystems in a many-qubit system, and this was later shown to be equivalent, for pure states, to the averaged linear entropies $\left(1-\pi_{A}\right)$ of the subsystems [28]. The concept was generalized to arbitrary $k$-particle subsystems of manyqudit systems [29], where, for any particular subsystem $(A)$, one averages over all distinct pairs of associated states in $B$,

$$
E_{A}=\sum_{a<a^{\prime}} \mathcal{W}^{2}\left(a, a^{\prime}\right)
$$

One may easily verify that $E_{A}$ is equivalent to the above measures by noting first that the unrestricted sum over all $a$ and $a^{\prime}$ gives simply $2 E_{A}$, because the $\mathcal{W}(a, a)$ terms vanish. The 
double sums are now done trivially using Equations 13 , demonstrating the identities

$$
2 E_{A}=1-\pi_{A}=c^{2}
$$

so that minimal $\pi_{A}$ values correspond to maximal $E_{A}$ as well as maximal $\mathcal{C}$ values.

\section{STEERING BY PROJECTIVE MEASUREMENTS}

As is well-known, all graph states are maximally connected, in the sense that any two particles can be projected into a Bell state by appropriate measurements on the others [1]. Not every set of measurements succeeds, however; some produce product states. In this section we trace the outcomes of all single measurements and all measurement pairs, comparing the persistency of entanglement as well as the flexibility for producing threeparticle GHZ states with one measurement, and Bell states with two. Again we assume that $d$ is a prime, where the existence of complete sets of mutually unbiased bases (MUBs) [30] makes the analysis and the results more compact.

To introduce the analysis, suppose we prepare the pure four-particle state $\left|\psi^{(4)}\right\rangle$, and perform a measurement on particle 1 of the observable $U$ [31] (henceforth we will write $U_{1}$ when we wish to specify which particle). If the outcome of the measurement is $\omega^{i}$ $(i=0,1, \ldots, d-1)$, then particle 1 is found in the state which we shall call $|U(i)\rangle$, while particles 2, 3, and 4 are projected into the associated pure three-particle state

$$
\left|\psi^{(3)}\right\rangle \sim\left\langle U_{1}(i) \mid \psi^{(4)}\right\rangle
$$

where $(\sim)$ is used because one may wish to regard $\left|\psi^{(3)}\right\rangle$ as normalized while the right side is not [its norm squared is the probability of the measurement outcome $\left(\omega^{i}\right)$ ].

Expressing these pure states as density matrices, $\rho^{(\mu)}=\left|\psi^{(\mu)}\right\rangle\left\langle\psi^{(\mu)}\right|$, Eq. 24 becomes

$$
\rho^{(3)} \sim\left\langle U_{1}(i)\left|\rho^{(4)}\right| U_{1}(i)\right\rangle
$$

The state $\rho^{(4)}$ may be expanded as a sum of its stabilizers, so that the projected (pure) states, $\rho^{(3)}$ and then $\rho^{(2)}$, are identified by their expansions [32]. The stabilizers form groups which are generated by multiplication among any four independent elements, called the generators, $g_{1}, \ldots, g_{4}$. The stabilizers are thus given by $S\left(p_{1}, p_{2}, p_{3}, p_{4}\right)=g_{1}^{p_{1}} g_{2}^{p_{2}} g_{3}^{p_{3}} g_{4}^{p_{4}}[33$, 
TABLE II: Generator sets for $G, C$ and $P$ states, (a) as taken directly from the graphs, and (b) as Fourier transformed with the states $G^{\prime}, C^{\prime}$, and $P^{\prime}$ of Eqs. 6- 8 .

\begin{tabular}{|c|ccc|ccc|}
\hline & \multicolumn{3}{|c|}{ (a) } & \multicolumn{3}{c|}{ (b) } \\
\hline & $G$ & $C$ & $P$ & $G^{\prime}$ & $C^{\prime}$ & $P^{\prime}$ \\
\hline$g_{1}$ & $X Z Z Z$ & $X Z I Z$ & $X Z I Z$ & $X X X X$ & $X X I X$ & $X X I X$ \\
$g_{2}$ & $Z X I I$ & $Z X Z I$ & $Z X Z^{-1} I$ & $Z Z^{-1} I I$ & $Z Z^{-1} Z I$ & $Z Z^{-1} Z^{-1} I$ \\
$g_{3}$ & $Z I X I$ & $I Z X Z$ & $I Z^{-1} X Z$ & $Z I Z^{-1} I$ & $I X X X$ & $I X^{-1} X X$ \\
$g_{4}$ & $Z I I X$ & $Z I Z X$ & $Z I Z X$ & $Z I I Z^{-1}$ & $Z I Z Z^{-1}$ & $Z I Z Z^{-1}$ \\
\hline
\end{tabular}

where $p_{n}=0,1, . ., d-1$, etc., producing a total of $d^{4}$ stabilizers including the identity. So the stabilizer expansion takes the form of a power series in the generators,

$$
\rho^{(4)}=d^{-4} \sum_{p_{1}, p_{2}, p_{3}, p_{4}}\left(e_{1}^{*} g_{1}\right)^{p_{1}}\left(e_{2}^{*} g_{2}\right)^{p_{2}}\left(e_{3}^{*} g_{3}\right)^{p_{3}}\left(e_{4}^{*} g_{4}\right)^{p_{4}}
$$

where the complex quantities, $e_{1}, \ldots, e_{4}$, are the eigenvalues of the corresponding generators, $g_{1}, \ldots, g_{4}$. This relation is derived in Appendix A for interested readers, but one may confirm immediately that $\rho^{(4)}$ is in fact an eigenstate of each generator $g_{n}$ with eigenvalue $e_{n}$.

The virtue of this approach is that, if we only care about the type of state and not the particular state itself, we need only specify the four generators of $\rho^{(4)}$. From these, we may deduce three generators for $\rho^{(3)}$, and from these in turn, two generators for $\rho^{(2)}$. These generator sets alone determine the type of state and the nature of its entanglement.

Let us briefly describe the generator sets for $\rho^{(4)}$ and then proceed with the analysis. The generator sets inferred directly from the graphs are listed in Table II(a): Each generator, $g_{n}$, identified with its single $X$ factor (implicitly $X_{n}$ ), is associated with the vertex $n$. The other factors are powers of $Z$ associated with adjacent vertices - those connected by edges. The power of $Z$ is dictated by the edge color: $Z$ with blue, and $Z^{-1}$ with red. (These rules are expressed more formally in Appendix A.) Table II(b) shows Fourier transformed generator sets compatible with the simplified forms of the graph states (Eqs. 6- 8). These transformations amount to the replacements $Z \rightarrow X$ and $X \rightarrow Z^{-1}$, which are applied in the $G$ case to particles 2,3 , and 4 ; and in the $C$ and $P$ cases to particles 2 and 4 . One may easily confirm that the states written in Eqs. 6- 8 are indeed joint eigenstates of the four corresponding generators, each with eigenvalue unity. 
One can see that the full stabilizer sets, as produced by any of the generator sets listed in Table II, contain elements in which an arbitrary one-body operator $\left(U=X^{n} Z^{m}\right)$ appears as the factor associated with any qudit. That is, writing the stabilizers as tensor products, $S=\sigma_{1} \sigma_{2} \sigma_{3} \sigma_{4}$, some stabilizers will have $\sigma_{1}=U$, others will have $\sigma_{2}=U$, and so forth. If we measure $U_{1}$, for example, then all those stabilizers having $\sigma_{1}=U$ (or a power $U^{k}$ ), will survive the projection in Eq. 25, with coefficients $\left(\omega^{i k}\right)$ depending on the measured value $\left(\omega^{i}\right)$. All other stabilizers will be annihilated by the projection, because the expectation value of any one-body operator $(V)$ in an eigenstate of another ( $U$, not a power of $V$ ) vanishes when the corresponding one-particle bases are mutually unbiased [20, 30]:

$$
\langle U(i)|V| U(i)\rangle=\sum_{j=0}^{d-1}\langle U(i) \mid V(j)\rangle \omega^{j}\langle V(j) \mid U(i)\rangle=\frac{1}{d} \sum_{j=0}^{d-1} \omega^{j}=0 .
$$

In the following examples, we shall consider measurements by all distinct one-body operators (excluding their powers, which would be redundant). Thus, the measured operator $U$ will be $X, Z$, or $\left(X Z^{k}\right)$, where $k=1,2, \ldots, d-1$; a total of $d+1$ choices for each particle. With each input state to follow, we consider the outcomes of all such first measurements, and then (conditionally) of all possible second measurements.

\section{A. GHZ states}

Suppose that $\rho^{(4)}$ is any GHZ state expanded in the generators of Table II(b); the choice of eigenvalues is immaterial. Consider $X$ measurements. Because $G$ states are symmetric under particle permutations, the results will not depend on the choice of particle measured, and so for convenience we choose $X_{4}$. To determine which stabilizers survive the projection of Eq. 25 and contribute to $\rho^{(3)}$, it suffices to look at just the generators: Clearly the first three survive (because powers of $X$ include the identity), leaving $X X X, Z Z^{-1} I$, and $Z I Z^{-1}$. These three form a commuting generator set for the stabilizers that make up $\rho^{(3)}$, and we recognize it as a GHZ set [34]. Thus, we know that $\rho^{(3)}$ is a $G$ state without carrying the analysis further. As a check, measuring $X$ on another particle $(m)$ produces the same outcome, because products among $g_{2}, g_{3}$, and $g_{4}$ produce identity factors $I_{m}$ on the same particle. Next consider $Z$ measurements: If we measure $Z_{1}$, the projection (25) produces

inverses of $Z I I, I Z I$, and $I I Z$, which qualify as generators for $\rho^{(3)}$. These three indicate a product state and the removal of all entanglement. Clearly the same outcome results from 
a $Z$ measurement on any other particle. Finally, consider the remaining cases, $U=\left(X Z^{k}\right)$ $(k \neq 0)$. We can generate stabilizers that associate the factor $\mathrm{U}$ with any particle we like; for example, $g_{1} g_{2}^{-k}$ associates it with the fourth. A measurement of $U_{4}$ then projects from this $\left(X Z^{-k}\right) X X$, in addition to $Z Z^{-1} I$, and $Z I Z^{-1}$, which form a generator set for $\rho^{(3)}$. Clearly the measurement of $U$ on any other particle $(m)$ projects a similar set of operators, one containing $\left(X_{m} Z_{m}^{-k}\right)$ as a factor among $X \mathrm{~s}$, the others containing factors of $Z, Z^{-1}$, and I. Clearly these comprise GHZ generator sets. The upshot is that all one-particle measurements, with the exception of $Z$ measurements, produce three-particle GHZ states; the $Z$ measurements produce product states. To put this in representation-free terms, GHZ entanglement is vulnerable to one out of the $d+1$ possible measurement bases.

This vulnerability remains in place for the second measurement, because the operators $Z Z^{-1} I$, and $Z I Z^{-1}$ are always present in the expansion of $\rho^{(3)}$, whatever the first measurement was. As a result, a second measurement of $Z_{n}$ on any qudit $n$ will project (among others) the operators $Z I$ and $I Z$, which qualify as generators for $\rho^{(2)}$ and identify a product state. All other measurement choices, $X$ or $V=\left(X Z^{l}\right) \quad(l=1, \ldots, d-1)$, will project $Z Z^{-1}$ and a second operator of the form $\left(X Z^{-i}\right)\left(X Z^{-j}\right)$, where $i$ and $j$ may take the values $0, k$, or $l$, depending on what the first measurement was. These two generators produce (generalized) Bell states [2, 3], the basic example being

$$
|\psi\rangle=\frac{1}{\sqrt{d}} \sum_{i}|i, i\rangle .
$$

One may verify that indeed this is an eigenstate of the two generators above, whatever specific value the second generator takes (if the second generator is $X X$, for example, then the eigenvalue is unity).

Putting together the above outcomes: Out of all first measurements, a fraction $d /(d+1)$ project three-particle GHZ states. Out of all second measurements on these, the same fraction $d /(d+1)$ project Bell states, their two-particle analogs. So $d^{2} /(d+1)^{2}$ of all combined measurements produce Bell states. The remaining fraction, $(2 d+1) /(d+1)^{2}$, produce product states. These outcomes are summarized on Table III. 
TABLE III: (a) Distribution of first-measurement outcomes resulting from the $d+1$ distinct measurements on each particle, reflecting single vulnerable bases for the $G$ and $C$ states, and (b) distribution of two-measurement outcomes resulting from $(d+1)$ second measurements on each remaining particle [total number of measurement sequences is $12(d+1)^{2}$ ].

(a) first measurements

\begin{tabular}{|c||c|c|c|}
\hline input & $\pi^{(3)}$ & $S_{n} B$ & $G^{(3)}$ \\
\hline$G$ & 4 & 0 & $4 d$ \\
$C$ & 0 & 4 & $4 d$ \\
$P$ & 0 & 0 & $4(d+1)$ \\
\hline
\end{tabular}

(b) measurement pairs

\begin{tabular}{|c||c|c|}
\hline input & $\pi^{(2)}$ & $B$ \\
\hline$G$ & $24 d+12$ & $12 d^{2}$ \\
$C$ & $20 d+8$ & $12 d^{2}+4 d+4$ \\
$P$ & $12 d+12$ & $12 d^{2}+12 d$ \\
\hline
\end{tabular}

\section{B. $\mathbf{P}$ states}

The analysis for $P$ states is the simplest because, although it is not immediately obvious from the generators, there is no stabilizer with more than a single $I$ factor. This point was made in Ref. [5], but we give a concise proof here in Appendix C. As a consequence, the state $\rho^{(3)}$ resulting from any Pauli measurement, on any qudit, consists of stabilizers with the same property, and is therefore nonseparable [35]. The only nonseparable 3-particle joint eigenstates of Pauli operators are GHZ states [5]. Therefore, remarkably, every first measurement choice projects a 3-particle GHZ state.

Since all second measurements must be on GHZ states, then, according to the arguments given above, $d /(d+1)$ of these project Bell states, while the remaining fraction, $1 /(d+1)$ project product states. These fractions are reflected in Table III(b).

Remarkably, however, there is no a priori basis of vulnerability for $P$ states. Rather, the basis vulnerable to second measurements is determined by the basis chosen for the first measurement, and also the choice of qudit. These conditional vulnerabilities arise from the 
variety of GHZ states projected by first measurements: Every basis appears as the vulnerable one as the result of some first measurement.

\section{Cluster states}

Cluster entanglement has less a priori vulnerability to measurement bases than GHZ entanglement, but such vulnerability exists and it is more complicated. We examine the special cases where this enters by considering two revealing stabilizers, each of which contains two $I$ factors, namely $g_{2} g_{4}^{-1}=I Z^{-1} I Z$ and $g_{1} g_{3}^{-1}=X I X^{-1} I$. These identify $Z$ as the vulnerable basis for particles 2 and 4 , while $X$ is vulnerable for 1 and 3. Specifically, a measurement of $Z_{2}$ projects $I I Z$ and $X X^{-1} I$ from the two stabilizers above, and $Z Z I$ from $g_{2}$ itself. This means that particles 1 and 3 are projected into a Bell state, leaving particle 4 in a pure eigenstate of $Z$. This type of state is labelled $S_{4} B$ [5], identifying the separated particle. By similar arguments, a measurement of $Z_{4}$ produces a state of type $S_{2} B$. The corresponding vulnerable basis for particles 1 and 3 is $X$ : A measurement of $X_{3}$ projects $X I I$ and $I Z^{-1} Z$ from the above stabilizers, and $I X X$ from $g_{3}$ itself. Thus, the projected state is of the type $S_{1} B$. A measurement of $X_{1}$ projects a state of type $S_{3} B$. In all cases, the separating particle is diagonally opposed to the measured particle on the square graph. These are the four special cases of enhanced vulnerability to second measurements. It is easy to show that all other first measurements, comprising a fraction $d /(d+1)$ of the total number, produce 3-particle GHZ states, as reflected on Table III.

When the first measurement projects a GHZ state, then, as in the previous cases, $d /(d+1)$ of the second measurements produce Bell states, while the remaining $1 /(d+1)$ produce product states. In the four remaining special cases involving $S_{n} B$ states, a measurement on either of the Bell state particles removes the entanglement, while a measurement on the separated particle preserves the Bell state of the other two. Comparing these vulnerable cases with those of GHZ states, rather than losing all entanglement to first measurements, we only make it more susceptible to second measurements, where it is lost in $2 / 3$ of the cases, but preserved in the remaining $1 / 3$. As a result, the fraction of successes is increased by $1 / 3(d+1)$ over that of the GHZ case, as reflected in Table III.

To illustrate how the combination of all measurement pathways leads to the final results,

Fig. 2 shows the trajectories taken by 48 distinct measurement pairs in the special case 

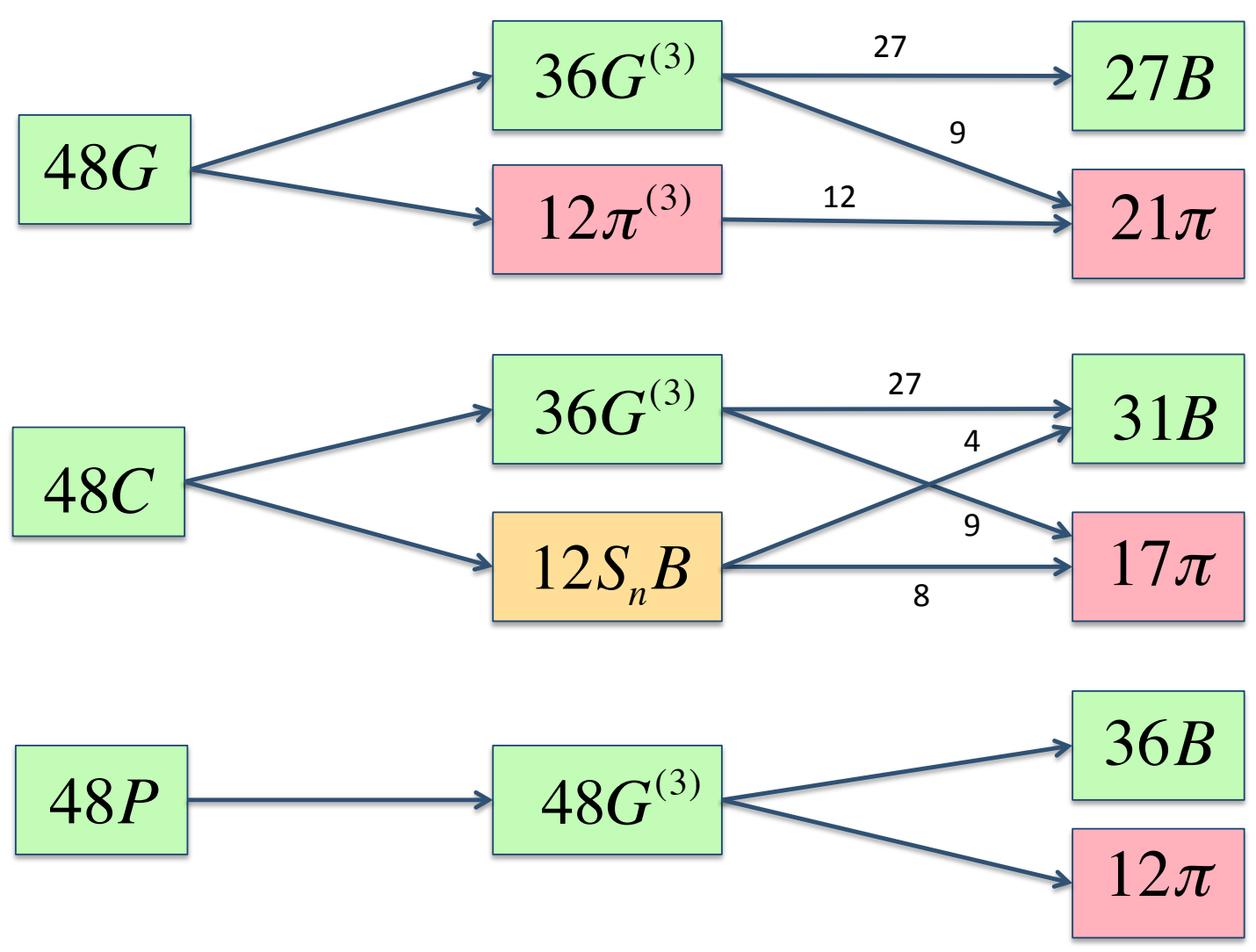

FIG. 2: Number of measurement paths per qutrit for each sequence of outcomes.

of $d=3$. There are actually $12(d+1)^{2}=192$ distinct ordered pairs, but the outcome statistics are independent of which qutrit is chosen for the first measurement, so the number is effectively reduced to 48 . The outcomes after two measurements show a steady increase in robustness of entanglement as we progress from $G$ to $C$ to $P$, as well as in steering flexibility for producing arbitrary Bell states.

\section{Generalized Pauli Persistency}

Recall that the simple Pauli persistency is the minimum number of measurements, $\mathcal{N}_{\text {min }}$, required to completely remove the entanglement of the system. One can define a generalized Pauli persistency as the number of measurements in an arbitrary sequence, $\mathcal{N}\left(U_{n}, V_{m}\right)$, after which the entanglement is completely removed. This number depends on the operators chosen for the first two measurements, but not on the third, which removes any remaining entanglement. In the case of the $C$ states, it also depends on the choice of the second qudit measured, given the first $(i e$, on $m-n)$. 
We define the average Pauli persistency over all measurement paths,

$$
\mathcal{N}_{\text {ave }}=\sum_{\text {paths }} \mathcal{N}\left(U_{n}, V_{m}\right)\left(\sum_{\text {paths }}\right)^{-1}
$$

Since the choice of third measurement is irrelevant, as is the choice of qudit for the first measurement, the average should be taken over a total of $3(d+1)^{2}$ paths, 3 being the number of choices of the second qudit. There are 48 such paths represented in Fig. 3 for qutrits. The averages over these paths are $\mathcal{N}_{\text {ave }}=2.31,2.65$, and 2.75 for the $G, C$, and $P$ states, respectively. As $d$ increases, these numbers all increase and approach the value 3 asymptotically as $d \rightarrow \infty$. Although these numbers distinguish between the three states in the expected order, they are not dramatic. The more interesting distinction is in the difference between successes and failures in the final outcomes after two measurements.

With $d=3$, for example, the normalized difference, $\Delta=[\mathcal{N}(B)-\mathcal{N}(\pi)] / 48$, takes the values $0.125,0.292$, and 0.50 , for $G, C$, and $P$, respectively.

\section{E. Comment on Reduced States of Subsystems}

In Sec. III we derived the reduced density matrices of all subsystems directly from the states. In Appendix $\mathrm{C}$ we recover these results in operator form (more concisely but with less insight), using the stabilizer formalism introduced here. The main point of this is to show that a four-particle graph state is a 2-MM state if and only if none of its stabilizers has more that a single $I$ factor, a property unique to the $P$ states.

\section{CONCLUSIONS AND OPEN QUESTIONS}

We have shown that in four-particle systems, unlike two- and three-particle systems, there is a qualitative jump in the potential for entanglement in going from binary to nonbinary cases. This is because a third entanglement class is introduced for all prime $d \geq 3$ in addition to the two that exist for qubits. We have documented the quantitative differences among the three classes in terms of the reduced states of all subsystems, as well as the persistency of entanglement under measurement-induced steering.

The subsystem states (and particularly their purities as listed in Table I) show that $N=4$ is a transitional case, in the sense that there is a threshhold dimension $(d=3)$ for 
the existence of maximally multiparticle entangled states (MMES): Only the $P$ states satisfy the requisite condition that all subsystems of up to half the system size are maximally mixed. In fact, $N=4$ is the smallest such transitional number, and we expect that there exist larger systems $(N)$ which are transitional in the same sense, whose threshhold dimensions could be larger than 3. Such transitions would open up new possibilities for maximal multiparticle entanglement, a property known to be inaccessible to systems of 8 or more qubits [7]. It remains an unanswered question whether (i) there exist MMES states of 7 or more particles of any $d$, and (ii) if so, then for some such $d$, what are the limits on $N$ for their existence?

Both aspects of our study, but particularly the steering analysis, highlight the extraordinary symmetries of the $P$ states with regard to permutations of both particles and measurement bases. A consequence of these symmetries is that the three-particle GHZ states projected by first measurements on $P$ states exhibit weak (or vulnerable) bases of all types (uniformly distributed over $Z$ and $X Z^{k}, k=0,1, \ldots, d-1$ ), while those projected from $G$ and $C$ states are biased toward $Z$ or $X$ as weak bases. Another general unanswered question is whether states with similarly high permutation symmetries will be found in higher-order transitional cases, exhibiting maximal entanglement at higher $N$ and $d$.

\section{Appendix A: Graphs, states and stabilizers}

Here we review the formalism that relates graphs to states and stabilizers through the so-called adjacency matrix, $\Gamma$, whose elements specify the edge weights: The edge connecting vertices $n$ and $m$ is assigned the weight $\Gamma_{n m}=0,1, \ldots, d-1$ (where 0 means no edge), so that diagonal elements are zero and $\Gamma_{n m}=\Gamma_{m n}$. In our examples, blue denotes the value 1 , and red denotes $d-1=-1$.) According to the rules described in the text, the stabilizer generators are given by

$$
g_{n}=X_{n} \bigotimes_{m} Z_{m}^{\Gamma_{n m}}
$$

and the stabilizers, labeled by the powers of the generators, are given by

$$
S\left(p_{1} p_{2} p_{3} p_{4}\right)=g_{1}^{p_{1}} g_{2}^{p_{2}} g_{3}^{p_{3}} g_{4}^{p_{4}}=\prod_{n}\left(X_{n} \bigotimes_{m} Z_{m}^{\Gamma_{n m}}\right)^{p_{n}}
$$


To re-express A2 as an overall tensor product, we must reorder the factors to bring those operating on the same qudit together,

$$
S\left(p_{1} p_{2} p_{3} p_{4}\right)=\omega^{\sum_{n>m} \Gamma_{n m} p_{n} p_{m}} \bigotimes_{n=1}^{4} X_{n}^{p_{n}} Z_{n}^{\sum_{m=1}^{4} \Gamma_{n m} p_{m}},
$$

where the phase prefactor results from the general commutators, $Z_{m}^{b} X_{n}^{a}=\omega^{a b} X_{n}^{a} Z_{m}^{b}$.

The graph states are given in terms of standard basis states (here written $\left|j_{1} j_{2} j_{3} j_{4}\right\rangle$ ) by

$$
|\psi\rangle=\sum_{j_{1} j_{2} j_{3} j_{4}}\left|j_{1} j_{2} j_{3} j_{4}\right\rangle \exp \left(\frac{2 \pi i}{d} \sum_{n=1}^{4} \Gamma_{n m} j_{n} j_{m}\right) .
$$

This is the joint eigenstate of all four generators with eigenvalues unity. In total, there are $d^{4}$ joint eigenstates with eigenvalues $\left(e_{1}, e_{2}, e_{3}, e_{4}\right) \equiv\left(\omega^{r_{1}}, \omega^{r_{2}}, \omega^{r_{3}}, \omega^{r_{4}}\right)$, where $r_{k}=0,1, \ldots, d-1$, and we label their density matrices by these powers, $\rho^{(4)}\left(r_{1} r_{2} r_{3} r_{4}\right)$.

The stabilizers A2 have eigenvalues $\omega^{r_{1} p_{1}+r_{2} p_{2}+r_{3} p_{3}+r_{4} p_{4}}$ in the states $\rho^{(4)}\left(r_{1} r_{2} r_{3} r_{4}\right)$, and so clearly their spectral representations are

$$
S\left(p_{1} p_{2} p_{3} p_{4}\right) \equiv \sum_{r_{1} r_{2} r_{3} r_{4}} \rho^{(4)}\left(r_{1} r_{2} r_{3} r_{4}\right) \omega^{r_{1} p_{1}+r_{2} p_{2}+r_{3} p_{3}+r_{4} p_{4}} .
$$

This is a four dimensional Fourier transform on a hypercube with $d$ points on a side. Its inverse is

$$
\rho^{(4)}\left(r_{1} r_{2} r_{3} r_{4}\right)=d^{-4} \sum_{p_{1} p_{2} p_{3} p_{4}} S\left(p_{1} p_{2} p_{3} p_{4}\right) \omega^{-r_{1} p_{1}-r_{2} p_{2}-r_{3} p_{3}-r_{4} p_{4}} .
$$

We immediately recover Eq. 26 of Sec. IV by writing $\mathrm{S}$ as the product of generators and recognizing the exponent as the product of $\left(e_{n}^{*}\right)^{p_{n}}$ factors.

\section{Appendix B: Completeness of four-particle $G, C$, and $P$ graphs for prime $d$}

Here we prove that an arbitrary four-particle graph is equivalent, under local unitary transformations and permutations of qudits, to one of the $G, C$, or $P$ types shown in Fig. 1, for prime $d$. Any such transformation on a graph state can be represented by combinations of two operations on its adjacency matrix $\Gamma$ [36]: The first, $\circ_{n}(f)$, consists of multiplying the $n$-th row and $n$-th column by $f \neq 0$,

$$
\circ_{n}(f) \Gamma_{l m} \equiv f^{\delta_{n l}+\delta_{n m}} \Gamma_{l m}
$$



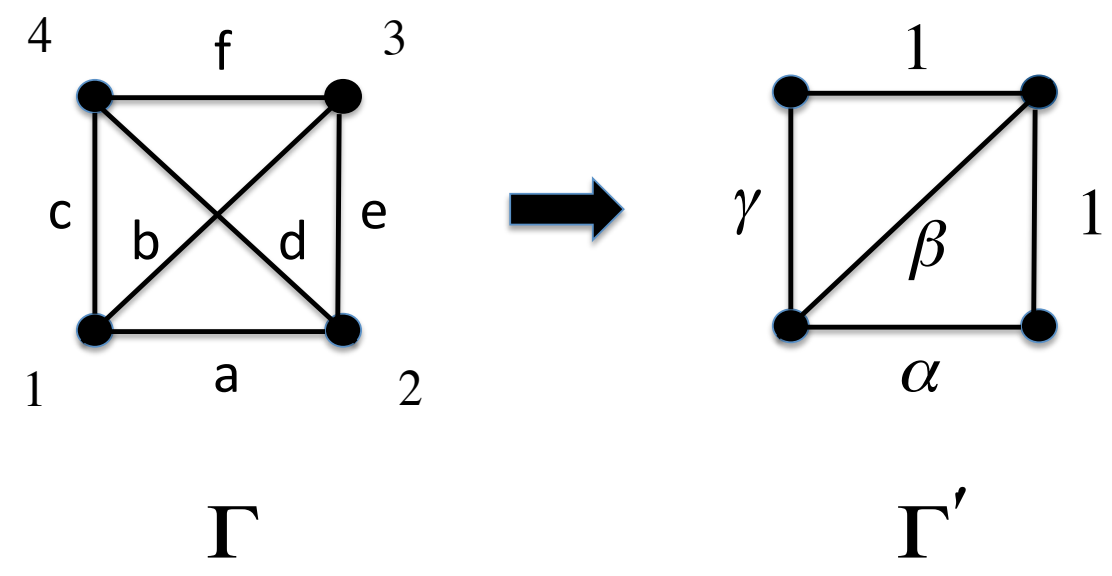

FIG. 3: Most general six-edged graph and its reduction to an equivalant five-edged graph.

while the second, $*_{n}(f)$, induces changes outside the $n$-th row and column,

$$
*_{n}(f) \Gamma_{l m}=\Gamma_{l m}+f \Gamma_{l n} \Gamma_{n m}
$$

Two graphs are equivalent if their $\Gamma$ matrices are related by a sequence of these two operations together with permutations of qudits (interchanges of corresponding rows and columns).

Let us begin with the general six-edged graph shown in Fig. 3, in which all edge weights are nonvanishing. The associated adjacency matrix is

$$
\Gamma=\left(\begin{array}{cccc}
0 & a & b & c \\
a & 0 & e & d \\
b & e & 0 & f \\
c & d & f & 0
\end{array}\right)
$$

We can remove any element [say $d$, by the operation $*_{3}\left(-d e^{-1} f^{-1}\right)$ ] and scale two of the remaining elements to unity [say $o_{3}\left(e^{-1}\right)$ then $\circ_{4}\left(e f^{-1}\right)$ ], leaving the five-edged graph,

$$
\Gamma^{\prime}=\left(\begin{array}{cccc}
0 & \alpha & \beta & \gamma \\
\alpha & 0 & 1 & 0 \\
\beta & 1 & 0 & 1 \\
\gamma & 0 & 1 & 0
\end{array}\right)
$$

where the operations above dictate that

$$
\alpha=a-b d f^{-1}
$$




$$
\begin{gathered}
\beta=b e^{-1}, \\
\gamma=(c e-b d) f^{-1} .
\end{gathered}
$$

Note that either or both of $\alpha$ and $\gamma$ may vanish without any of the original parameters vanishing. If both vanish, $\alpha=\gamma=0$, then the permutation $(3 \leftrightarrow 4)$ and scaling $\circ_{1}\left(\beta^{-1}\right)$ reduce $\Gamma^{\prime}$ to

$$
\Gamma_{G}=\left(\begin{array}{llll}
0 & 0 & 0 & 1 \\
0 & 0 & 0 & 1 \\
0 & 0 & 0 & 1 \\
1 & 1 & 1 & 0
\end{array}\right)
$$

which is equivalent to the GHZ graph in Fig. 1. Now suppose that at least one of $\alpha$ and $\gamma$ is nonzero. Since they are interchangeable (by $2 \leftrightarrow 4$ ) choose $\underline{\alpha \neq 0}$ : Then the operation $*_{2}\left(-\beta \alpha^{-1}\right)$ removes $\beta$, and the scaling $\circ_{1}\left(\alpha^{-1}\right)$ leaves

$$
\Gamma(\tilde{\gamma})=\left(\begin{array}{cccc}
0 & 1 & 0 & \tilde{\gamma} \\
1 & 0 & 1 & 0 \\
0 & 1 & 0 & 1 \\
\tilde{\gamma} & 0 & 1 & 0
\end{array}\right),
$$

where

$$
\tilde{\gamma}=\gamma / \alpha=(c e-b d) /(a f-b d)
$$

which is well-defined because $\alpha \neq 0$. Clearly $\tilde{\gamma}=1$ corresponds to the $C$ graph of Fig. 1 .

We can show immediately that the three-sided graph associated with $\tilde{\gamma}=0$ is equivalent to this: Its state is given by Eq. 2 with the phase factor changed to $\omega^{j(i+k)+l k}$. The $j$ and $l$ sums can be identified as Fourier transforms as before, but now the resulting state is

$$
\left|C^{\prime \prime}\right\rangle=\frac{1}{d} \sum_{i, k}|i, i+k, k, k\rangle .
$$

Comparing with the $\left|C^{\prime}\right\rangle$ expression (7), both have repeated indices, but in different places. So the permutation $(2 \leftrightarrow 3)$ followed by simple variable changes maps Eq. B9 into 7 .

All remaining cases correspond to the remaining values of $\tilde{\gamma}$, namely $2, \ldots, d-1$. One can see immediately that the $\tilde{\gamma}=-1$ case, with permutations $1 \leftrightarrow 2$ and $3 \leftrightarrow 4$, corresponds to the $P$ state defined by Fig. 1. Appendix $\mathrm{C}$ shows formally that all of the values, 
$\tilde{\gamma}=2, \ldots, d-1$, have the same entanglement characteristics, by calculating the reduced density matrices as functions of $\tilde{\gamma}$. One reaches the same conclusion with the approach of Sec. III, using the general form (Eq. 9 ) for $C$ and $P$ states (repeated here):

$$
|\psi(\tilde{\gamma})\rangle=\frac{1}{d} \sum_{i, k}|i, i+\tilde{\gamma} k, k, i+k\rangle .
$$

For maximal mixing of all pairs (the 2-MM property) we require that the basis states in $A$ span $\mathcal{H}_{A}$, and that their associated states span $\mathcal{H}_{B}$, for any choice of the pair, $A=n, m$. This can happen if and only if $\tilde{\gamma}=2, \ldots, d-1$ and $d$ is a prime. The other values, $\tilde{\gamma}=0$ and 1 , allow repeated indices which cause one of the pairings to fail.

We have shown thus far that an arbitrary six-edged graph must reduce to one of the $G$, $C$, or $P$ classes. This proof incorporates some, but not all, lower-edged graphs. Here we sketch proofs that cover them all.

(i) Five-edged graphs: All six placements of the 0 element are equivalent under permutations $n \leftrightarrow m$, so choose $\Gamma_{24} \equiv d=0$ (in the notation of B3). Then remove $b$ using $*_{2}(-b / a e$ ) and scale $a, e$, and $f$ to unity. This leaves $\Gamma(\tilde{\gamma})$ in the form of Eq. B7, with $\tilde{\gamma}=c e / a f$ nonzero, which is equivalent to a $C$ or $P$ state depending on the value of $\tilde{\gamma}$. (Note that no five-edged graph can reduce to a GHZ state.)

(ii) Four-edged graphs: There are 15 ways to place two zeroes in the matrix. In three of these, the zeroes are diagonally-coordinated. These three are equivalent under permutations alone, and one of them is $\Gamma(\tilde{\gamma})$ with $\tilde{\gamma}$ nonzero. The remaining 12 graphs are identical among themselves under permutations, so choose $c=d=0$. Again remove $b$ using $*_{2}(-b / a e)$ and scale the remaining three elements to unity, leaving $\Gamma(\tilde{\gamma})$, now with $\tilde{\gamma}=0$, a $C$ state. (Note that no four-edged graph reduces to a GHZ state.)

(iii) Three-edged graphs: Since there are 20 ways to place three zeroes in the matrix, let us focus instead on the graphs themselves. Clearly there are four GHZ graphs in which a single vertex connects to three edges. These four are related by cyclic permutations, or rotations of Fig. 1a. It is easy to see (without enumerating) that all remaining graphs must have two vertices each connected to two edges, and two vertices each connected to single edges. The vertices can be rearranged and aligned so that the edges form a straight line without overlapping. Starting with this line, one may count 16 ways of placing the vertices back on a square (making four distinct shapes, each with four orientations). All 16 are permutation-equivalents to $\Gamma(\tilde{\gamma})$ with $\tilde{\gamma}=0$, a $C$ state. 


\section{Appendix C: Reduced density matrices - stabilizer expansions}

Here we rederive the reduced density matrices of Sec III from the stabilizer formalism and obtain the results in purely operator form. We begin with the pure four-particle density matrix as given by Eqs. A3 and A6, with eigenvalues taken as unity,

$$
\rho^{(4)}=d^{-4} \sum_{p_{1} p_{2} p_{3} p_{4}} \omega^{\sum_{n>m} \Gamma_{n m} p_{n} p_{m}} \bigotimes_{n=1}^{4} X_{n}^{p_{n}} Z_{n}^{\sum_{m=1}^{4} \Gamma_{n m} p_{m}} .
$$

Specializing first to the $G$ state with $\Gamma_{G}$ given by Eq. B6, this density matrix (letting $\left.p_{1} \ldots p_{4} \rightarrow a, b, c, d\right)$ becomes

$$
\rho(G)=d^{-4} \sum_{a b c d} \omega^{(a+b+c) d}\left(X^{a} Z^{d}\right) \otimes\left(X^{b} Z^{d}\right) \otimes\left(X^{c} Z^{d}\right) \otimes\left(X^{d} Z^{a+b+c}\right) .
$$

Tracing over any particle would give an equivalent result, so choose $n=4$; surviving terms have $d=0$ and $a+b+c=0$, so that

$$
\operatorname{Tr}_{4}[\rho(G)]=d^{-3} \sum_{a b} X^{a} \otimes X^{b} \otimes X^{-a-b}
$$

The choice of second particle is again immaterial, so choose $n=3$; the result is

$$
\rho_{1,2}=\operatorname{Tr}_{3,4}[\rho(G)]=d^{-2} \sum_{a} X^{a} \otimes X^{-a}
$$

This shows that no two-particle subsystem is maximally mixed in the $G$ state.

We now treat the $C$ and $P$ states simultaneously using $\Gamma(\tilde{\gamma})$ of Eq. B7. The density matrix (henceforth replacing $\tilde{\gamma} \rightarrow \gamma$ ) is

$$
\rho(\gamma)=d^{-4} \sum_{a b c d} \omega^{a b+b c+c d+\gamma a d}\left(X^{a} Z^{b+\gamma d}\right) \otimes\left(X^{b} Z^{a+c}\right) \otimes\left(X^{c} Z^{b+d}\right) \otimes\left(X^{d} Z^{\gamma a+c}\right)
$$

Tracing over the fourth particle removes all but the $d=0$ and $c=-\gamma a$ terms, resulting in

$$
\operatorname{Tr}_{4}[\rho(\gamma)]=d^{-3} \sum_{a b} \omega^{(1-\gamma) a b}\left(X^{a} Z^{b}\right) \otimes\left(X^{b} Z^{(1-\gamma) a}\right) \otimes\left(X^{-\gamma a} Z^{b}\right)
$$

The choice of second particle now matters, and we must treat three(!) cases separately:

(i) Tracing over particle 1 (the simplest case, with $a=b=0$ ) gives us

$$
\rho_{2,3}=\operatorname{Tr}_{1,4}[\rho(\gamma)]=d^{-2} I \otimes I
$$

showing that the subsystem $(2,3)$ is maximally mixed. 
(ii) Tracing instead over particle 2, we get

$$
\rho_{1,3}=\operatorname{Tr}_{2,4}[\rho(\gamma)]=d^{-2} \sum_{d} X^{a} \otimes X^{-\gamma a} \delta_{(1-\gamma) a, 0}
$$

This reduces to $d^{-2} I \otimes I$ unless $\gamma=1$ (the four-sided $C$ state).

(iii) Tracing finally over particle 3 gives us

$$
\rho_{1,2}=\operatorname{Tr}_{3,4}[\rho(\gamma)]=d^{-2} \sum_{d} X^{a} \otimes Z^{(1-\gamma) a} \delta_{\gamma a, 0},
$$

which also reduces to $d^{-2} I \otimes I$, but now unless $\gamma=0$ (the three-sided $C$ state). Cases (i-iii) taken together show that all two-particle subsystems are maximally mixed for the $P$-states, $\gamma=2, \ldots, d-1$, while for $\gamma=0$ or 1 ( $C$ states $)$, only two of the three pairs are maximally mixed.

These outcomes may be traced to the fact that no $P$ stabilizer has more than a single $I$ factor (proved immediately below), while some $C$ stabilizers do: For $\gamma=1$, the $I$ factors are diagonally opposed (case ii), while for $\gamma=0$ they appear together, on qudits 1,2 in one instance and on 3,4 in the other (case iii). In the case of $G$ states, there are stabilizers in which $I$ factors appear on an arbitrary pair of qudits, so that no subsystem of two particles is maximally mixed.

\section{Proof that $P$ stabilizers have at most single $I$ factors}

The generators dictated by $\Gamma(\tilde{\gamma})$ of Eq. B7 (again letting $\tilde{\gamma} \rightarrow \gamma$ ) are

$$
X Z I Z^{\gamma}, \quad Z X Z I, \quad I Z X Z, \quad Z^{\gamma} I Z X
$$

The resulting stabilizers (ignoring the commutation-generated phase factors) are

$$
S(a, b, c, d)=\left(X^{a} Z^{b+\gamma d}\right) \otimes\left(X^{b} Z^{a+c}\right) \otimes\left(X^{c} Z^{b+d}\right) \otimes\left(X^{d} Z^{\gamma a+c}\right) .
$$

For comparison, let us first identify $C$-state stabilizers with more than single $I$ factors:

(i) If $\gamma=0$, then $g_{1}=S(1,0,0,0)$ and $g_{4}=S(0,0,0,1)$ each have two $I$ factors.

(ii) If $\gamma=1$, then $g_{1} g_{3}^{-1}=S(1,0,-1,0)$ and $g_{2} g_{4}^{-1}=S(0,1,0,-1)$ each have two $I$ factors. Now for the $P$ states:

(iii) If $\gamma$ takes any other value $(2, \ldots, d-1)$, then one can write out each of six possible

conditions for the existence of at least two $I$ factors, and show immediately that none of these has a nontrivial solution. 
This proves that no $P$-state stabilizer has more than a single $I$ factor. This property is a necessary and sufficient condition for $P$ states to have the 2-MM property, because then and only then, tracing over the states of any two particles annihilates all of the stabilizers, so that only the identity $I \otimes I$ survives.

[1] H.-J. Briegel and R. Raussendorf, Phys. Rev. Letters 86, 910 (2001).

[2] T. Durt, e-print arXiv:quant-ph/0401046; also see discussion on pp. 43-46 of Ref. [4].

[3] A.B. Klimov, D. Sych, L.L. Sánchez-Soto, and G. Leuchs, Phys. Rev. A 79, 052101 (2009).

[4] T. Durt, B.-G. Englert, I. Bengtsson, and K. Zyczkowski, Int. J. Quantum Inf. 08, 535 (2010).

[5] J. Lawrence, Phys. Rev. A 84, 022338 (2011).

[6] P. Facchi, G. Florio, G. Parisi, and S. Pascazio, Phys. Rev. A 77, 060304(R) (2008).

[7] L. Arnaud and N.J. Cerf, Phys. Rev. A 87, 012319 (2013).

[8] M. Hein, W. Dür, J. Eisert, R. Raussendorf, M. Van den Nest, and H.J. Briegel, in Quantum Computers, Algorithms and Chaos, Vol. 162 Int. School of Physics Enrico Fermi (eds. G. Casati et. al.) 115-218 (IOS Press, Amsterdam, 2006).

[9] R. Raussendorf and H.-J. Briegel, Phys. Rev. Letters, 86, 5188 (2001).

[10] H.-J. Briegel, D.E. Browne, W. Dür, R. Raussendorf, and M. Van den Nest, Nature Physics 5, 19 (2009).

[11] J.L. Romero, G. Björk, A.B. Klimov, and L.L. Sánchez-Soto, Phys. Rev. A 72, 062310 (2005).

[12] M. Wieśniak, T. Paterek, and A. Zeilinger, New J. Phys. 13, 053047 (2011).

[13] D. Bruss and C. Macchiavello, Phys. Rev. Letters 88, 127901 (2002).

[14] P.K. Aravind, Z. Naturforsch. 58a, 682 (2003).

[15] T. Durt, N.J. Cerf, N. Gisin, and M. Zukowski, Phys. Rev. A 67, 012311 (2003), generalized the Ekert protocol [37] to qutrits.

[16] S. Gröblacher, T. Jennewein, A. Varizi, G. Weihs, and A. Zeilinger, New Journal of Phys. 8, 75 (2006), reported an experimental demonstration of a variation of the Ekert protocol [38] using qutrits.

[17] A. Ashikhmin and E. Knill, IEEE Trans. Info. Theory, 47, 3065 (2001).

[18] M. Bahramgiri and S. Beigi, e-print arXiv:quant-ph/0610267 (2006).

[19] D. Hu, W. Tang, M. Zhao, Q. Chen, S. Yu, and C.H. Oh, Phys. Rev. A 78, 012306 (2008). 
[20] S. Bandyopadhyay, P.O. Boykin, V. Roychowdhury, and F. Vatan, Algorithmica 34, 512 (2002).

[21] For a recent general discussion, see J. Sperling and W. Vogel, Phys. Scr. 83, 045002 (2011).

[22] I. Sainz and G. Björk, Phys. Rev. A. 76, 042313 (2007).

[23] S. Hill and W.K. Wootters, Phys. Rev. Letters 78, 5022 (1997).

[24] W.K. Wootters, Phys. Rev. Letters 80, 2245 (1998).

[25] A. Uhlmann, Phys, Rev. A 62, 032307 (2000).

[26] P. Rungta, V. Buzek, C.M. Caves, M. Hillery, and G.J. Milburn, Phys. Rev. A 64, 042315 (2001). See in particular Eq. (18).

[27] D.A. Meyer and N.R. Wallach, J. Math. Phys. 43, 4273 (22002).

[28] G.K. Brennen, Quantum Inf. Comp. 3, 619 (2003).

[29] A.J. Scott, Phys. Rev. A 69, 052330 (2004).

[30] W.K. Wootters and B.D. Fields, Ann. Phys. 191, 363 (1989); and in Bell's Theorem, Quantum Theory and Conceptions of the Universe, edited by M. Kafatos (Kluwer Academic Publishers, Dordrecht, 1989), pp. 65-67.

[31] We refer to $U$ and other Pauli factors as observables, with the understanding that, being unitary, they are exponentials of hermitian operators which have real eigenvalues, $a=0,1, \ldots, d-1$, the eigenvalues of $U$ being $\omega^{a}$.

[32] See Sec. III(E) of Ref. [18] for a general discussion.

[33] Our notation is similar to that of D.H. Zhang, H. Fan, D. L. Zhou, Phys. Rev. A, 79, 042318 (2009), (Sec. II).

[34] One can see that this generator set is related by Fourier transforms to that defined by the three-qudit GHZ graph. Alternatively, one may verify immediately that the three-particle analog of Eq. 6 is an eigenstate of this generator set.

[35] One can easily show that a separable three-particle eigenstate of Pauli operators must have stabilizers with two $I$ factors [5]. Nontrivial examples are found in the case of C-states, to follow.

[36] M. Bahramgiri and S. Beigi, e-print arXiv:cs/0702057v2 (2007).

[37] A.K. Ekert, Phys. Rev. Letters, 67, 661 (1991).

[38] T. Jennewein, C. Simon, G. Weihs, H. Weinfurter, and A. Zeilinger, PRL 84, 4729 (2000). 